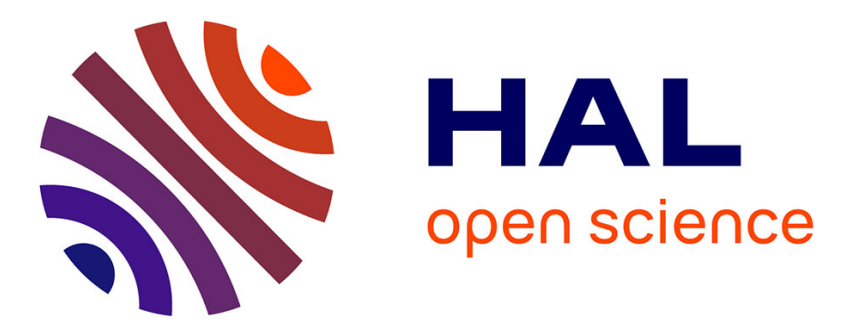

\title{
Sustainability analysis: Viability concepts to consider transient and asymptotical dynamics in socio-ecological tourism-based systems
}

Wei Wei, Isabelle Alvarez, Sophie Martin

\section{- To cite this version:}

Wei Wei, Isabelle Alvarez, Sophie Martin. Sustainability analysis: Viability concepts to consider transient and asymptotical dynamics in socio-ecological tourism-based systems. Ecological Modelling, 2013, 251, pp.103-113. 10.1016/j.ecolmodel.2012.10.009 . hal-01170511

HAL Id: hal-01170511

https://hal.science/hal-01170511

Submitted on 5 Apr 2017

HAL is a multi-disciplinary open access archive for the deposit and dissemination of scientific research documents, whether they are published or not. The documents may come from teaching and research institutions in France or abroad, or from public or private research centers.
L'archive ouverte pluridisciplinaire HAL, est destinée au dépôt et à la diffusion de documents scientifiques de niveau recherche, publiés ou non, émanant des établissements d'enseignement et de recherche français ou étrangers, des laboratoires publics ou privés. 


\title{
Sustainability analysis: Viability concepts to consider transient and asymptotical dynamics in socio-ecological tourism-based systems
}

\author{
W. Wei ${ }^{\mathrm{a}}$, I. Alvarez ${ }^{\mathrm{a}, \mathrm{b}}$, S. Martin ${ }^{\mathrm{a}, *}$ \\ ${ }^{a}$ Irstea, LISC - Clermont-Ferrand, 24 av. des Landais, BP 50085, 63172 Aubière Cedex, \\ France \\ ${ }^{b}$ Université de Paris VI, LIP 6, B.P. 169, 4 place Jussieu, 75252 Paris Cedex 05, France
}

\section{Abstract}

With the increasing pressure on natural resources, the sustainability study of socio-ecological systems has become a crucial scientific issue. In this paper we emphasize that transient behaviors have to be taken into account in sustainability analysis. We illustrate their impact with a model of tourism development from the literature, where the sustainability study is based on asymptotic properties. In order to evaluate relevant space and time characteristics of transient dynamics, we propose to use the concepts and tools of the mathematical viability theory. We also extend this analysis to controlled dynamical systems, which are particularly appropriate to model socio-ecological systems when management issues are at stake.

Keywords: Sustainable development, Dynamical system, Asymptotic analysis, Viability kernel, Capture basin.

\footnotetext{
${ }^{*}$ Corresponding author

Email addresses: wei.wei@irstea.fr (W. Wei), isabelle.alvarez@irstea.fr (I. Alvarez), sophie.martin@irstea.fr (S. Martin)
} 


\section{Introduction}

In 1987, the Brundtland Report (Brundtland, 1987) gave the definition of sustainable development as development "which meets the needs of the present without compromising the ability of future generations to meet their own needs". Since then this concept has been widely studied. This is especially perceptible in the field of Economics (see Munasinghe, 1993), the social sphere (Jackson, 2007; Lehtonen, 2004) and the environmental field (Gunderson and Holling, 2002). Facing the vagueness of the notion of sustainability (Levin, 1993), most of these works lead to make more explicit the relation between sustainability and other concepts such as stability, resilience, robustness or risk (see Ludwig et al., 1997; Perrings, 2006).

In the case of theoretical approaches, a dynamical system is generally used to model the time dependency of the different state variables of the system. When the model consists of a set of differential equations, sustainability and related concepts are often linked to asymptotic properties: The value and the properties of the attractors (related for instance to the value of the eigenvalues), properties in the phase space (for example, the size of attractor capture basin (Collings and Wollkind, 1990; Coller, 1997), and properties of the bifurcation diagram (see for instance Ludwig et al., 1997; Casagrandi and Rinaldi, 2002; Lacitignola et al., 2007)). In the bifurcation diagram parameters can be seen as slow variables compared to the state space variables.

However, as stated in Chichilnisky (1997), it is not possible to forget the present state when dealing with sustainability, so asymptotic properties are not sufficient to characterize sustainability, since they focus on the far future. But appropriate representations are difficult to assess. It is the case 
in economics, where classical discounted utility seems to favor present states (Chichilnisky, 1996). Other criteria, such as maximin (Solow (1974); Cairns and Van Long (2006)), which maximizes the minimal utility over time, have been proposed in that concern.

Moreover, most natural systems never reach any equilibrium, since they are submitted to frequent perturbations. When multiple attractors coexist, they can even switch after a perturbation from one attraction basin to another (May, 1977). Therefore, it can be worthy to take into account some characteristics of transient behaviors. Transient behaviors can have undesirable impacts on the system, leading to states that can be very far from the final attractor, and the time required to reach a satisfactory neighborhood of the attractor can also be very long. In fact, this information is important to study resilience (Martin, 2004), which is an important component to understand sustainability (Neubert and Caswell, 1997). This paper proposes to use constraints in the state space to address the issue of transient behaviors. Such a use of constraints as guardrails has already been proposed for climate change studies through the concept of Tolerable Windows (PetschelHeld et al., 1999). The Safe Minimum Standard approach introduced by Ciriacy-Wantrup (1952) is also based on constraint specification.

In order to take into account constraints of desirable states and related characteristics of transient behavior, we propose to use the mathematical viability theory (Aubin, 1991), since it develops methods and tools to study the compatibility between dynamics and constraints. This framework has been used by Bruckner et al. (2003) to describe the Tolerable Windows approach. Moreover, using as constraint set the set of desirable states allows addressing 
sustainability issue as the possibility of finding a path that is an acceptable compromise for all parties (Fuentes, 1993). Such an approach has already been proposed in Martinet and Doyen (2007) considering an economy with an exhaustible resource or in Martinet et al. (2007) and in Chapel et al. (2008) to determine sustainable fisheries.

In section II, we describe two limits of the asymptotic stability analysis: First, the time factor due to transient dynamics. Even in very simple configurations, transient dynamics can lead to unacceptable delay to reach a given neighborhood of the attractor. Second, the space factor. In many situations it is not desirable to see the system move far away from the attractor, which can occur during transient dynamics. The maximal distance to the final attractor along the evolution can represent an unacceptable condition in a more or less distant future. We illustrate these issues using a model of tourism development similar to the model from Casagrandi and Rinaldi (2002), because it can exhibit interesting behaviours (several attractors including either equilibria or limit cycles). In section III, we first show that when defining appropriate constraint sets, spatial and temporal lacking information can be captured by the concepts of viability kernel and capture basin. We illustrate how the limits pointed out in section II can be over passed using these viability concepts. In section IV we discuss the viability approach for controlled dynamics, when some parameters can be interpreted as control variables. Actually, controlled dynamics are often appropriate to represent dynamics of socio-ecological systems facing sustainability issues influenced by human management decision or behavior (see for instance Melbourne-Thomas et al., 2011; Costamagna and Landis, 2006). In the concluding section we consider 
further use of viability theory to address sustainability issues.

\section{Limits of the asymptotic stability analysis viewpoint}

In this section, we consider that a model based on a set of differential equations is available. This is often the case when studying sustainability from a theoretical viewpoint. As an example, we illustrate our argument with a model of tourism impact on environment quality, the asymptotic analysis of which was performed in Casagrandi and Rinaldi (2002).

\subsection{Overview of the model of tourism impact on environment quality}

The model described in Casagrandi and Rinaldi (2002) deals with the problem of the interaction between tourism and environment, at a very abstract level. It is three-dimensional with twelve parameters. Ten parameters are fixed and the asymptotic analysis is performed according to the two remaining ones. The model (1) studies the interactions between the tourist activity $T(t)$ in the area at time $t$, the quality of the natural environment $E(t)$ and the capital $C(t)$ intended as structures for tourist activities.

$$
\begin{aligned}
\dot{T}(t) & =\frac{d T(t)}{d t}=T(t)\left[\mu_{E} \frac{E(t)}{E(t)+\varphi_{E}}+\mu_{C} \frac{C(t)}{C(t)+\varphi_{C} T(t)+\varphi_{C}}-\alpha T(t)-a\right] \\
\dot{E}(t) & =\frac{d E(t)}{d t}=E(t)\left[r E(t)\left(1-\frac{E(t)}{K}\right)-\beta C(t)-\gamma T(t)\right] \\
\dot{C}(t) & =\frac{d C(t)}{d t}=-\delta C(t)+\epsilon T(t)
\end{aligned}
$$

$\dot{T}(t)$ is denoted by $f_{1}(t), \dot{E}(t)$ by $f_{2}(t)$ and $\dot{C}(t)$ by $f_{3}(t)$ in the following. First equation describes the variation of the tourist activity, which is proportional to the present tourist activity and the relative attractiveness of the site. The attractiveness consists of the sum of two positive factors, the attractiveness of the environment and the attractiveness of the infrastructure, 
and two negative factors, the linearly decreasing congestion and the average value of the attractiveness of all tourist sites ( $a$ can be seen as a measure of the competition exerted by alternative tourist sites). Fixed parameters are $\mu_{E}$, the attractiveness associated with high environmental quality, and $\varphi_{E}$ the half saturation constant (the environmental quality at which tourist satisfaction is half maximum). $\mu_{C}$ and $\varphi_{C}$ are the corresponding parameters for the attractiveness of the infrastructure. Congestion is proportional to $T$ with factor $-\alpha$.

Second equation describes the variation of environment quality. It consists first of a logistic equation which describes the impact on the environment of all activities except tourism industry. Parameters $r$ and $K$ are the net growth rate and the carrying capacity of the logistic function. The two other terms represent the flow of damages induced by tourism. Generally, this flow is positively correlated with tourist activity and capital with factors $\gamma$ and $\beta$.

Last equation describes the rate of change of capital as the difference between the investment flow $\epsilon T$ and the depreciation $\delta C$ ( $\epsilon$ is the investment rate and $\delta$ the depreciation rate).

The main assumption of the model is that parameter $\delta$ is supposed to be small compared with $r$ to take into account the fact that the degradation of tourist structures is very slow.

We use in this paper the same parameter values as in Casagrandi and Rinaldi (2002): $r=K=\alpha=\beta=\gamma=\varphi_{C}=1, \delta=0.1, \varphi_{E}=0.5, \mu_{E}=\mu_{C}=$ 10.

In Casagrandi and Rinaldi (2002), the two varying parameters of the 
asymptotic analysis are $a$ which measures the competition exerted by alternative tourist sites and $\epsilon$ the investment rate.

This relatively simple model reproduces fairly Butler's scenarios (Butler, 1980) for tourist sites development. Figure 1 displays for instance three scenarios of tourism evolution with the same parameter values $(a=6)$ except for the investment rate $\epsilon$ which takes three different values $(\epsilon=0.01,0.1$ and 0.45$)$. The initial point of all curves is $(T=0.01, E=1, C=0.01)$ but with three different values of the investment rate $\epsilon$ during 100 time units, one scenario leads to tourist activity disappearance and the two others lead to non null tourist activity but with different levels.

\subsection{Information provided by asymptotic study and bifurcation diagram}

Asymptotic analysis focuses on the infinite time horizon behavior of the system. For instance, for the three scenarios described in figure 1, asymptotic analysis allows predicting toward which values the model variables converge. These values are necessarily equilibria of the dynamics, but there may also exist limit cycles. Such information is obviously valuable in a sustainability viewpoint which underlines future awareness. In the general case of an evolutionary system described by a differential equation

$$
x^{\prime}(t)=f(x(t))
$$

with $x \in \mathbb{R}^{n}$ the $n$-dimensional state variable vector and $f: \mathbb{R}^{n} \rightarrow \mathbb{R}^{n}$ Lipschitz continuous $^{1}$, an equilibrium point $x_{0} \in \mathbb{R}^{n}$ is a point where the

\footnotetext{
${ }^{1}$ The Lipschitz continuous condition ensures the local existence and uniqueness of the solution.
} 
dynamics is null $\left(f\left(x_{0}\right)=0\right)$. Nevertheless, this equilibrium may be asymptotically stable if it tends to attract the states in its vicinity, or unstable if some points in its vicinity tend to be rejected by it.

Equilibrium values are determined by solving the equation $f\left(x_{0}\right)=0$ analytically if possible or with well-known approximation methods (NewtonRaphson, fix point, gradient descent algorithm, etc).

Then, to determine the stability of these equilibria, we can use the linearization of the dynamics at these equilibrium points:

Theorem 1. Let $f$ differentiable and $A=\left.\frac{\partial f(x)}{\partial x}\right|_{x=x_{0}}$ be the Jacobian matrix of $f(x)$ with respect to $x$ evaluated at the equilibrium point $x_{0}$. Then the system

$$
z^{\prime}=A z
$$

is referred to as the linearization of equation (2), about the equilibrium point $x_{0}$. When the linearization exists, its stability determines the local stability of the original nonlinear equation.

Hence, if the real part of all the eigenvalues of $A$ are strictly negative, then the equilibrium is stable.

From model (1), obviously, $(\bar{T}=0, \bar{E}=0, \bar{C}=0),(\bar{T}=0, \bar{E}=K$, $\bar{C}=0)$ and also $(\bar{T}=\tilde{T}, \bar{E}=0, \bar{C}=\tilde{C})$ for some strictly positive values of $\tilde{T}$ and $\tilde{C}$, are equilibria of the dynamics. However, as it is done in Casagrandi and Rinaldi (2002), we emphasize the equilibria with strictly positive values for $T$ and $E$ : Casagrandi and Rinaldi (2002) define as "profitable" the attractor whereby tourist activity is maintained (but environment may be damaged), as "compatible" the attractor whereby the complete degradation 
of environment is avoided (but tourism may disappear) and as "sustainable attractor" the attractor whereby economic activities and environment preservation coexist. A sustainable equilibrium is then defined by $(\bar{T}>0, \bar{E}>0)$. A sustainable attractor is defined by $(\forall t, T(t)>0, E(t)>0)$.

Sustainable equilibria verify:

$$
\begin{aligned}
& \mu_{E} \frac{\bar{E}}{\bar{E}+\varphi_{E}}+\mu_{C} \frac{\bar{C}}{\bar{C}+\varphi_{C} \bar{T}+\varphi_{C}}=\alpha \bar{T}+a \\
& \bar{T}=\frac{r \bar{E}\left(1-\frac{\bar{E}}{K}\right)}{\frac{\beta \epsilon}{\delta}+\gamma} \\
& \bar{C}=\frac{\epsilon}{\delta} \bar{T}
\end{aligned}
$$

We used Newton-Raphson method (as described in Bruck et al., 1989) to approximate the strictly positive equilibrium values of model $(1),(\bar{T}>0$, $\bar{E}>0, \bar{C}>0$ ), for the parameter values used in the scenarios displayed in figure 1. For the three different values of the investment rate $\epsilon$ used in the three scenarios of figure 1, we obtain

- when $\epsilon=0.01,(\bar{T} \approx 0.167, \bar{E} \approx 0.758, \bar{C} \approx 0.017)$,

- when $\epsilon=0.1,(\bar{T} \approx 0.125, \bar{E} \approx 0.526, \bar{C} \approx 0.125)$,

- and when $\epsilon=0.45,(\bar{T} \approx 0.044, \bar{E} \approx 0.402, \bar{C} \approx 0.197)$.

Comparing these calculations and the simulations displayed in figure 1, we can notice that for the scenarios with $\epsilon=0.01$ and $\epsilon=0.1$, tourist activity seems to converge to the strictly positive equilibrium. On the contrary, for the scenario with $\epsilon=0.45$, tourist activity seems to converge to 0 whereas the value of the strictly positive equilibrium is approximatively 0.044 .

To go further and determine the stability of these strictly positive equilibria, we first determine the Jacobian matrix $M(\bar{T}, \bar{E}, \bar{C})$ of model dynamics 
at strictly positive equilibrium points. We will then calculate the eigenvalues of these matrices.

$$
\begin{gathered}
M(\bar{T}, \bar{E}, \bar{C})=\left(\begin{array}{ccc}
\frac{\partial f_{1}}{\partial T}(\bar{T}, \bar{E}, \bar{C}) & \frac{\partial f_{1}}{\partial E}(\bar{T}, \bar{E}, \bar{C}) & \frac{\partial f_{1}}{\partial C}(\bar{T}, \bar{E}, \bar{C}) \\
\frac{\partial f_{2}}{\partial T}(\bar{T}, \bar{E}, \bar{C}) & \frac{\partial f_{2}}{\partial E}(\bar{T}, \bar{E}, \bar{C}) & \frac{\partial f_{2}}{\partial C}(\bar{T}, \bar{E}, \bar{C}) \\
\frac{\partial f_{3}}{\partial T}(\bar{T}, \bar{E}, \bar{C}) & \frac{\partial f_{3}}{\partial E}(\bar{T}, \bar{E}, \bar{C}) & \frac{\partial f_{3}}{\partial C}(\bar{T}, \bar{E}, \bar{C})
\end{array}\right) \\
=\left(\begin{array}{ccc}
\bar{T}\left(\frac{-10 \bar{C}}{(\bar{C}+\bar{T}+1)^{2}}-1\right) & \bar{T}\left(\frac{5}{(\bar{E}+0.5)^{2}}\right) & \bar{T}\left(\frac{10 \bar{T}+10}{(\bar{C}+\bar{T}+1)^{2}}\right) \\
\bar{E} & \bar{E}(1-2 \bar{E}) & -\bar{E} \\
\epsilon & 0 & -0.1
\end{array}\right)
\end{gathered}
$$

We now calculate the eigenvalues for the different matrices $M(\bar{T}, \bar{E}, \bar{C})$ with software Scilab. We obtain the following rounded values:

- $\{-0.289+0.612 i,-0.289-0.612 i,-0.100\}$ for $\epsilon=0.01$,

- $\{-0.044+0.478 i,-0.044-0.478 i,-0.263\}$ for $\epsilon=0.1$,

- and $\{0.009+0.443 i,-0.009-0.443 i,-0.320\}$ for $\epsilon=0.45$.

Thanks to theorem 1, we can determine that when $\epsilon$ equals 0.01 and 0.1 , the strictly positive equilibrium is stable, because the real part of all the eigenvalues of the Jacobian matrix are negative. On the opposite for $\epsilon=0.45$, the strictly positive equilibrium is unstable. This result is entirely consistent with figure 1 where scenarios for $\epsilon$ equal to 0.01 and 0.1 seem to converge to the strictly positive equilibrium, whereas scenario for $\epsilon$ equal to 0.45 does not (it converges toward $(0,0,0)$ instead).

This study shows that when the investment rate is relatively low a sustainable equilibrium $(\bar{T}>0, \bar{E}>0$ ) exists (for instance for $\epsilon=0.01$ and $\epsilon=0.1)$ and is asymptotically stable. However, the tourist activity at this 
equilibrium point decreases with $\epsilon$. Moreover, it becomes instable for higher values of $\epsilon$ (e.g. $\epsilon=0.45)$.

Casagrandi and Rinaldi (2002) go further by determining the whole bifurcation diagram of model (1) in the parameter space $(\epsilon, a)$ : Each point of the two-dimensional space $(\epsilon, a)$ is associated with one specific set of attractors. They consider different values for the investment rate $\epsilon$ but also for the competition term from alternative tourist sites, $a$. The bifurcation curves partition the parameter space into subregions. All models corresponding to the same subregion have qualitatively the same long-term behavior, because they have the same kind of attractors. Then, Casagrandi and Rinaldi (2002) consider as safe situations where the only attractor is sustainable (strictly positive) and as risky, situations where both the strictly positive sustainable attractor and the equilibrium with $\bar{T}=0$ or $\bar{E}=0$ are stable because an unexpected accidental shock can perturb the state of the system and cause a jump ending to an attractor characterized by no tourism industry or a complete degradation of the environment.

\subsection{Missing information}

Evolutions toward stable attractors, whether risky or not from the bifurcation viewpoint, can nevertheless be undesirable because of their transient behavior.

\subsubsection{Spatial information: Attraction basin boundaries}

The attraction basin of an attractor gathers the states from which the evolution governed by system (2) is such that the distance to the attractor tends toward 0 . When there are several attractors, given an initial condition, 
the attraction basins of the different attractors are needed to determine the associated attractor.

For instance in model (1), when parameter values are $\epsilon=0.1$ and $a=$ 6.8 , the strictly positive equilibrium $(\bar{T}, \bar{E}, \bar{C})$ is no longer the only stable equilibrium: ( $T=0, E=1, C=0)$ is also a stable equilibrium. If the starting point is $(0.1,1,0.1)$, the evolution converges to the strictly positive equilibrium, but if the starting point is $(0.5,0.5,0.5)$, the evolution converges to the non strictly positive one (see Figure 2).

Consequently, the existence of a stable strictly positive equilibrium is not enough to assess the asymptotic behavior of any evolution. The computation of the attraction basin is also needed.

\subsubsection{Spatial information: Maximal distance to the attractor along the evo- lution}

Knowing that a given starting point belongs to the attraction basin of a particular attractor, a stable equilibrium for instance, may lead to believe that the distance from the evolving state of the system and the equilibrium will decrease with time. This extrapolation is not necessary right. Actually, the distance to the attractor is necessary bounded. But the distance to the attractor can vary a lot with time, and even increase, during the transient phase.

For instance, figure 3(a) displays two evolutions of the tourist activity for parameter values such that the unique attractor is the sustainable equilibrium (with $\bar{T} \approx 0.125$ ). Only the values of environment and capital at the initial point differ. For one evolution, the distance to the attractor is really decreasing (figure 3(b)), so the maximal distance to the attractor is the ini- 
tial one. On the contrary, for the second evolution, the maximal distance is reached approximatively at time 80 , where the tourist activity is more than twice the attractor value. Before that, during a relatively long time (approximatively between time 10 and 60), there is almost no tourist activity. At that time, the distance to the attractor is close to the attractor value. Knowing that the maximal distance to the attractor is much smaller than the attractor value would ensure that the tourist activity never decreases below a certain lower bound, or never suffers high variations.

Finding sustainable attractors, with respect to the coexistence of tourist activity and environment preservation, does not imply that any evolution will remain close to these attractors and exhibit good transient scenarios. Hence, the additive information of the maximal distance to the attractor along the evolution (or an upper bound) is necessary to ensure that bad situations are avoided.

Furthermore, there may be no stable equilibrium but a stable limit cycle as it is the case when $a=6$ and $\epsilon=0.13$. However, the cycles then performed by an evolution may belong to desirable states: It is the case of the scenario described by figure 4 , from time 20 , when the desirable states for the tourist activity are included between 0.02 and 0.30 (light gray area). On the contrary, when the desirable states are between 0.1 and 0.2 (dark gray area), then the evolution leading to this limit cycle should not be considered as acceptable.

\subsubsection{Temporal information: Time to reach a given neighborhood of the at- tractor}

A stable equilibrium is not reached in finite time except if the starting point is the equilibrium itself (as soon as $f$ is Lipschitz continuous, this 
is a consequence of the uniqueness of the solution). Nevertheless, if the initial condition belongs to its attraction basin, the time to reach a given neighborhood is finite.

This finite time may be short or much longer. Dotted curve of figure 1 is a typical curve which converges fast to stable equilibrium, but if we change the starting point to point $(T=0.26, E=1, C=0.26)$, the evolution displayed in figure 5, also converges to the stable point, but remains a long time $(20<t<300)$ very close to 0 . Such a scenario is unlikely to be acceptable. Actually, a long time with no tourist activity may not be considered as sustainable. Such a concern is taken into account in the sufficientarianism framework in which Chichilnisky (1977) proposed a criterion minimizing the time needed to reach an economic path that satisfies the basic needs, defining efficiency with respect to the minimization of the time horizon after which they are satisfied. However, this criterion does not take into account the possibility illustrated in the previous subsections and figure 3 that an evolution that satisfies the basic needs at time $t$ may not satisfy them anymore during a future time period. Conversely, a scenario may occur where tourism, environment and capital exhibit satisfactory values during a long time in the case of a single non-sustainable stable equilibrium. Depending on the time scale, such a scenario may be considered as desirable as we can imagine that the validity of the model will have to be questioned again in the future, or that other opportunities may occur before reaching undesirable states. 


\section{Viability concepts to provide missing information}

\subsection{Viability theory}

Viability theory concerns controlled dynamical systems. The evolution of the state variable vector $x \in X \subset \mathbb{R}^{n}$ is described by:

$$
\left\{\begin{array}{l}
x^{\prime}(t)=f(x(t), u(t)) \\
u(t) \in U(x(t))
\end{array}\right.
$$

where $U(x) \subset \mathbb{R}^{p}$ is the set of admissible controls when the state of the system is $x$.

Given a constraint set $K \subset X$, the viability theory methods and tools first aim at determining the viability kernel that is the subset of $K$ gathering all states of the system such that there exists at least one control function that allows the system to remain in the constraint set indefinitely:

$$
\operatorname{Viab}(K)=\{x \in K|\exists u(.)| x(t) \in K \forall t \in[0,+\infty[\}
$$

The states belonging to the viability kernel are called viable states. In the absence of control such as in differential equation model like model (1), the viability kernel gathers all starting points such that the evolution remains in the constraint set.

Another important concept of viability theory is the capture basin: Given a constraint set $K$ and a target set $C$, the capture basin gathers all states such that there exists a control function that allows reaching the target set while remaining in the constraint set.

$$
C a p t(K, C)=\{x \in K|\exists u(.), \exists T| x(T) \in C \text { and } x(t) \in K \forall t \in[0, T]\}
$$


We show in the two following subsections how these concepts of viability kernel and capture basin allow to obtain the missing information of the asymptotic analysis.

\subsection{Spatial information thanks to viability kernel}

In the case of the existence of a sustainable attractor $(\bar{T}>0, \bar{E}>0$, or $T(t)>0$ and $E(t)>0$ for all $t$, depending on the case), we showed in the previous section that one lacking information is the maximal distance to this attractor along the evolution. Viability kernel allows determining this maximal distance. Actually, if we consider a given neighborhood of this stable attractor, the viability kernel of this neighborhood gathers all states from which starts an evolution that remains in this neighborhood. Conversely, from any point outside this viability kernel, the evolution will go outside this neighborhood and the maximal distance to the attractor will be greater than its size.

We use again model (1) to provide an illustration. We take as parameter values $a=6$ and $\epsilon=0.1$. We have already proved in the previous section that the strictly positive attractor for these parameters value, $(\bar{T} \approx 0.125, \bar{E} \approx$ $0.526, \bar{C} \approx 0.125$ ), is stable. Let define a ball (for the sup norm) around this attractor with parameter $\Delta$ :

$$
K:=[\bar{T}-\Delta ; \bar{T}+\Delta] \times[\bar{E}-\Delta ; \bar{E}+\Delta] \times[\bar{C}-\Delta ; \bar{C}+\Delta] .
$$

This ball is considered as a constraint set in the viability theory framework and we approximate its viability kernel according to model (1) for different values of $\Delta$. This means that the value of the tourist activity and of the 
environmental quality should remain close to the attractor values. The state of the system is not allowed to drift outside the constraint set. In the following, we approximate viability kernels and capture basins using the algorithm described in Deffuant et al. (2007).

If we set for instance $\Delta=0.075$, the viability kernel is not the whole ball as displayed in figure 6 (a). The volume of viability kernel represents about $25 \%$ of the ball volume. For some points of the viability kernel, the norm sup distance to the attractor equals $\Delta$. On the contrary, for the many starting points outside the viability kernel (75\% of the ball volume), every evolution will leave the ball. So the maximal distance to the equilibrium along these evolutions will be necessarily greater than $\Delta$ in the future. Nevertheless some of these initial points are close to the attractor.

If we consider smaller balls, the viability kernels gather evolutions that remain closer to the equilibrium point. The proportion of the viability kernel volume compared to the ball volume remains almost constant around $24 \%$ (figure 6 (b) and figure 6 (c) display viability kernels for $\Delta$ values equal to 0.05 and 0.025 ). In figure 1 , the dotted curve makes oscillations before coming close to the attractor. The amplitude of these oscillations gets smaller and smaller with time. But, when we reduce the ball radius, those oscillations keep exceeding the constraints at the beginning of the evolution. For instance, for a tourist site, it can be undesirable to have a tourist activity that varies by a factor of two every three time units at the beginning of its development. That shows the importance of spatial information.

The viability kernel of the ball of radius $\Delta$ around the attractor gathers all points from which the evolution remains at a distance of the attractor 
smaller than $\Delta$. We then call these points $(\Delta$-) secure. The boundary of this kernel is then the $\Delta$ level set of the maximal distance to the attractor. Consequently, computing viability kernels with different values of $\Delta$ allows to approximate the graph of the maximal distance function.

We now choose a slightly greater value for $\epsilon, \epsilon=0.13$, which corresponds to a slightly higher investment rate in model (1). We know from the asymptotic analysis of the previous section that there is no stable equilibrium anymore but a limit cycle (figure 4). Nevertheless, whereas it is no more an equilibrium of the dynamics, the point $(\bar{T} \approx 0.125, \bar{E} \approx 0.526, \bar{C} \approx 0.125)$ remains a desirable situation with strictly positive values for the three variables. The question of determining the maximal distance to this point along an evolution remains then valuable in a sustainability perspective.

Figures 7 (a), 7 (b) and 7 (c) display the viability kernels for the same three constraint sets as the previous paragraph but with $\epsilon=0.13$ in the dynamics. The first two figures exhibit non-empty viability kernel. Hence, there exist evolutions remaining in the constraint set. Since all the evolutions converge toward the unique attractor which is the limit cycle, that implies that the limit cycle is included in the ball centered at $(\bar{T} \approx 0.125, \bar{E} \approx$ $0.526, \bar{C} \approx 0.125)$ with radius $\Delta=0.075$ and $\Delta=0.05$. On the contrary, when $\Delta=0.025$, the viability kernel is empty, which means that this smaller ball does not contain the limit cycle.

Hence, if desirable situations may be described by the ball centered at $(\bar{T} \approx 0.125, \bar{E} \approx 0.526, \bar{C} \approx 0.125)$ with $\Delta=0.025$, model $(1)$ does not produce any desirable evolution from any starting point: Actually, the viability kernel of this ball is empty, which means that from any starting point, the 
evolution leaves it in finite time. However, if desirable situations may be described by a bigger ball with radius $\Delta=0.05$ for instance, there exists numerous starting points leading to desirable evolutions even if there is no stable equilibrium but a limit cycle.

\subsection{Temporal information thanks to capture basin}

Belonging to a sustainable equilibrium attraction domain ensures that from such a starting point, the evolution will converge to this equilibrium. However, we have seen in subsection 2.3.3 that the time needed to reach a given neighborhood of this equilibrium may vary tremendously according to the starting point. This information may be provided by the capture basin of the viability theory considering this neighborhood as a target set.

Henceforth, desirable situations are described by a ball around the sustainable attractor. The viability kernel of this ball gathers the secure states, since from any starting point in this viability kernel, we are sure that the evolution will remain in the ball. The computation of the capture basins then allows to evaluate the time needed to reach a secure situation in the viability kernel of the set of desirable states. Such recovery issue has already been dealt with by Doyen and Saint-Pierre (1997) when they define the notion of minimum time of crisis (which measures the time spent by an evolution outside a given constraint set in the state space) used, for instance, by Béné et al. (2001) to analyze overexploitation of marine renewable resources or by Martinet et al. (2010) to analyze recovery paths for bioeconomic resource systems facing crisis situations.

Figure 8 (a) reproduces the viability kernel of previous section 3.2 dis-

played in figure 6 (c). Then, the eight following figures (Figure 8) display 
the capture basins of this viability kernel for eight increasing reaching time $(t=10, t=20, \ldots, t=80)$. From the figure, we can notice that points belonging to the capture basin are not equally distributed around the viability kernel, and only evolutions starting from initial points belonging to the capture basin displayed in figure 8 (i) reach the viability kernel with time smaller than 80. This observation highlights the fact that starting closer to the viability kernel and then closer to the equilibrium does not necessary imply reaching faster the viability kernel.

With such a measure, the effort to recover is evaluated by the time to reach secure situations. Depending on the application, more complex cost measures may appear more appropriate as proposed by Martin (2004) for the resilience evaluation. In the model of tourism development, for instance, a more complex cost function may be made up of two terms: The first term, which corresponds to the ecological cost, would measure the time spent with an environmental quality lower than the acceptable bound, $\underline{E}$; the second

one, which is an economic cost, would measure the time duration of the period of too low tourist activity weighted by the range of deviation from the acceptable lower bound, $\underline{T}-T(t)$.

\section{Viability concepts and sustainability analysis}

\subsection{Non controlled dynamics}

We have shown in the previous section, how two main concepts of viability theory, viability kernel and capture basin, can provide valuable information that complements asymptotic analysis. Given system dynamics, once asymptotic analysis has provided the asymptotically stable equilibria, it is 
possible to consider different neighborhoods centered around these attractors (like balls of different radius) and to compute the viability kernel of these neighborhoods. Viability kernels associate starting points with the maximal distance to the attractor. Capture basins of these viability kernels associate starting points with the time to reach (and remain in) a given neighborhood of the attractor. It is also possible to follow a slightly different approach. Given system dynamics, we can consider the subset of the state space that represents the set of desirable states, that is an acceptable compromise for all parties: For instance in the tourism model, $E \geq \underline{E}$ and $T \geq \underline{T}$ ensures that a given level of environmental quality is preserved as a certain level of tourist activity. The set of points from which the evolution remains in this desirable set is then a valuable information: This is the viability kernel of the desirable set considered as a constraint set; for the points outside the viability kernel, a valuable information is the time, maybe infinite, to reach this viability kernel, and this is the capture basin of the viability kernel.

In this viewpoint, the main concept is the set of desirable states, and not the asymptotic attractor: Different asymptotic behaviors can share the same set of desirable states, so bifurcations are not necessarily a problem, as long as new attractors are included in the same set of desirable states. Asymptotic analysis and viability viewpoint are clearly linked: If the desirable set does not contain any attractor, equilibrium nor limit cycle, then necessarily the viability kernel will be empty. But the viability paradigm centers sustainability on the definition of the desirable set, not on the asymptotic behavior of the system. Actually, the interest of the viability analysis is even more visible when controlled dynamics are involved, and this is the topic of next 
section.

\subsection{Controlled dynamics}

Sustainability problems often involve socio-ecological systems on which different action policies can be carried out, and the identification of efficient policies is by the way one crucial point.

Consequently, controlled dynamics are often more appropriate than noncontrolled dynamics to represent dynamics of systems facing sustainability and management issues.

In the tourism model we use as an illustration since the beginning of this article, the investment rate $\epsilon$ and the measure of the competition exerted by alternative tourist sites $a$ can for instance be considered as control variables on which a manager, a political power can act (for instance, by a management decision for the investment rate, and by advertising campaign for the competition control parameter). The system dynamics are then described by a controlled dynamical system:

$$
\left\{\begin{aligned}
\dot{T}(t) & =T(t)\left[\mu_{E} \frac{E(t)}{E(t)+\varphi_{E}}+\mu_{C} \frac{C(t)}{C(t)+\varphi_{C} T(t)+\varphi_{C}}-\alpha T(t)-a(t)\right] \\
\dot{E}(t) & =E(t)\left[r E(t)\left(1-\frac{E(t)}{K}\right)-\beta C(t)-\gamma T(t)\right] \\
\dot{C}(t) & =-\delta C(t)+\epsilon(t) T(t) \\
\epsilon(t) & \in[\underline{\epsilon} ; \bar{\epsilon}] \\
a(t) & \in[\underline{a} ; \bar{a}]
\end{aligned}\right.
$$

where $\underline{\epsilon}$ and $\bar{\epsilon}$ (resp. $\underline{a}$ and $\bar{a}$ ) are the bounds of the possible investment rates (resp. of the measure of the competition exerted by alternative tourist sites). In this second tourism model (6), $\epsilon$ and $a$ may vary with time and their 
evolutions are not defined beforehand. In the asymptotic analysis, the determination of the attractor needs the description of all variable evolutions with time. It is not the case in the viability framework: The desirable set can still be defined and the first noteworthy set gathers the starting points from which there exist control functions $t \rightarrow \epsilon(t)$ and $t \rightarrow a(t)$ that allow to keep the state of the system in the desirable set which is the viability kernel of the desirable set again; the second remarkable set gathers the starting points from which there exists control functions $t \rightarrow \epsilon(t)$ and $t \rightarrow a(t)$ such that the desirable set can be reached and then preserved which is the capture basin of the viability kernel again.

Hence, if the desirable set $K$ is defined by $T \in[0.1 ; 0.2], E \in\left[0.5 ; E_{\max }=\right.$ 0.6], $C \in[0.1 ; 0.2]$, computing the viability kernel with $\underline{\epsilon}=0.01, \bar{\epsilon}=0.3$, $\underline{a}=6$ and $\bar{a}=8$ allows determining the initial points such that there exist control functions that make the system state remain satisfactory along the evolution. Figure 9 displays this viability kernel.

From any starting point inside the viability kernel, there exists at least one control function that allows to keep the evolution in the constraint set. Such viable control functions are obtained as consequences of viability kernel computation. Figure 10 displays the trajectories of two evolutions starting at the same point: The black one is governed by a viable control function, so it remains in the constraint set, the points drawn on this trajectory correspond to positions where the control value changes; the gray one is obtained with fixed values for $\epsilon$ and $a$ and leaves the constraint set in finite time: At time $\mathrm{t}=39$, the value $C$ of the structures for tourist activities, falls below the minimum allowed by the constraint set. On the contrary, the controlled 
trajectory uses during the next 30 time units a higher value of the investment rate, in order to keep $C$ at a desirable level. Then the investment rate is set to an intermediate value, with some adjustment every 25 time units.

Besides, for this kind of tourism model, there is no reason to set an upper bound $E_{\max }$ for environment quality in the definition of the set of desirable states $K$. In the case of the model (6) with constant control $a=6$, we have found that when $E_{\max } \geq 0.65$, the viability kernel does not increase anymore (since other constraints are violated first).

Obviously, the viability kernel with controlled dynamics includes the viability kernels with constant controls, since control variations provide additional opportunities to find trajectories remaining in the constraint set.

Figure 11 presents two comparisons between the viability kernel of the controlled dynamics of model (6) with constant control $a=6$ and variable control $\epsilon \in[0.05,0.3]$ and two viability kernels obtained with fixed values of $\epsilon$. In both cases, the volume of the viability kernel of the controlled dynamics (in gray) is tree times bigger than the viability kernel with constant control (in black).

When non-controlled dynamics are concerned, the viability kernel allows distinguishing inside the constraint set points from which the evolution will remain in this constraint set from those which will leave it in finite time. In the case of controlled dynamics, the viability kernel separates points from which there exists at least one control function that governs an evolution which remains in the constraint set, from those from which the system will leave the constraint set in finite time whatever the control function. In this framework, points belonging to the viability kernel can be considered as sus- 
tainable on condition that a viable control function be applied, since the desirable system states represented by the constraint set can be preserved over time.

As in the non-controlled dynamics case, for points which do not belong to the viability kernel, a valuable information is the time needed to reach this viability kernel if possible. Actually, as long as the viability kernel is not reached, the system state is either already outside the constraint set or doomed to leave it (if it is still inside) whatever the control function.

In the controlled dynamics case, as in the constant control case, for a given reaching time, the capture basin of the viability kernel gathers all points from which there exists one control function that allows to reach the viability kernel in finite time smaller or equal to this reaching time. The boundary of the capture basin is then the level set of the minimum reaching time.

The minimum reaching time can then be used to define a measure of sustainability: The smaller it is, the more sustainable the state is, since the awkward period outside the viability kernel is shorter on condition that the right control function be applied. There may be initial points from which the viability kernel can not be reached whatever the control function. Such situations clearly can be considered as unsustainable.

\section{Conclusion and perspectives}

Asymptotic analysis together with bifurcation diagram provides useful information to understand the asymptotic behavior of a dynamical system. It identifies the attractors of the dynamics, equilibria or limit cycles, toward 
which the evolution of the model will converge eventually. It describes the change of attractors with the change of model parameters. We have shown, in this article, that asymptotic analysis lacks essential information for a sustainable analysis, using as an illustration a model of tourism impact on environment from Casagrandi and Rinaldi (2002). We have performed a stability analysis of the equilibria for different values of the investment parameter $(\epsilon)$. We have seen that, without information on the boundary of the attraction basin, it is not always possible to predict toward which attractor an evolution will converge. We have shown on examples that transient behavior can lead an evolution very far away from its attractor, much farther than the initial conditions, and this without considering any perturbations. We have also shown that it can take a finite but very long time for an evolution to reach a given neighborhood of its attractor. These behaviors can occur for attractors that are considered as sustainable and safe in the framework from Casagrandi and Rinaldi (2002).

In order to take into account these spatial and time factors of the transient dynamics in the sustainability analysis, we have introduced two main concepts of the viability theory: The viability kernel and the capture basin. Considering a set of desirable states around an attractor, the viability kernel gathers all the states in this neighborhood from which evolution always remains in the neighborhood. The capture basin gathers all the states from which the viability kernel is reached in a given time. As illustrations, we have computed viability kernels and capture basins for the tourism model. We have used these sets to compute several level sets of the maximum distance to the attractor and of the minimum reaching time (of a neighborhood 
of the attractor).

We have also explained how these concepts and the information they provide can be extended to controlled dynamics, which are more useful to represent socio-ecological systems when management issues are at stake. In the case of the tourism model, we have shown that controlled dynamics can be kept in the desirable constraint set more easily.

This work suggests a number of interesting research perspectives on sustainability in socio-ecological domain, when a dynamical system can be used to model the system behavior. The main interest of the method is to focus on the definition of a set of desirable states in which the stakeholders want to confine the system, rather than on the asymptotic attractor. It also suggests strongly identifying control possibilities among state variables and parameters, in order to model explicitly these control variables in the dynamical model. Viability theory and tools can then be used to compute the viability kernel and its capture basin, given a set of desirable states of the system, time constraints and admissible controls. Within the capture basin, there always exists a control strategy that will lead the system to the desirable set in acceptable time.

Further works from a methodological viewpoint includes comparison of different viability analyses, using different admissible control sets and different definitions of desirable states. Defining measures to compare the corresponding viability kernels, and defining the robustness of trajectories would allow comparing different control strategies. Moreover, we intend to take into account the effect of uncertainty in the definition of sustainable states: 
For instance, in the model of tourism development, the competition with the other tourist sites we consider as a control variable may be regarded as an uncertainty to which the tourist site under study is confronted. Such issues can be addressed in the viability theory framework using dynamic games (Aubin, 1997).

\section{References}

Aubin, J., 1991. Viability theory. Birkhäuser, Basel.

Aubin, J., 1997. Dynamic economic theory. Springer-Verlag, Berlin.

Béné, C., Doyen, L., Gabay, D., 2001. A viability analysis for a bio-economic model. Ecol. Econ. 36, 385-396.

Bruck, H., McNeill, S., Sutton, M., Peters, W., 1989. Digital image correlation using newton-raphson method of partial differential correction. Exp. Mech. 29, 261-267.

URL http://dx.doi.org/10.1007/BF02321405

Bruckner, T., Petschel-Held, G., Leimbach, M., Toth, F. L., 2003. Methodological aspects of the tolerable windows approach. Climatic Change 56, 73-89.

URL http://dx.doi.org/10.1023/A:1021388429866

Brundtland, G., 1987. Our Common Future [The Brundtland Report]. Oxford University Press, Oxford.

Butler, R. W., 1980. The concept of a tourist area cycle of evolution implication for management of resources. Can. Geogr. 8, 5-12. 
Cairns, R., Van Long, N., 2006. Maximin: A direct approach to sustainability. Environ. Dev. Econ. 11 (3), 275-300.

Casagrandi, R., Rinaldi, S., 2002. A theoretical approach to tourism sustainability. Conserv. Ecol. 6 (1), 13.

URL http://www.consecol.org/vol6/iss1/art13/

Chapel, L., Deffuant, G., Martin, S., Mullon, C., 2008. Defining yield policies in a viability approach. Ecol. Model. 212 (1-2), 10-15.

Chichilnisky, G., 1977. Economic development and efficiency criteria in the satisfaction of basic needs. Appl. Math. Model. 1, 290-298.

Chichilnisky, G., 1996. An axiomatic approach to sustainable development. Soc. Choice Welfare 13 (2), 231-257.

Chichilnisky, G., 1997. What is sustainable development? Land Econ. 73 (4), 467-491.

Ciriacy-Wantrup, S., 1952. Resource Conservation: Economics and Policies. University of California Press, Berkeley.

Coller, L. V., 1997. Automated techniques for the qualitative analysis of ecological models : continuous models. Conserv. Ecol. 1 (1), 5.

URL http://www. consecol.org/vol1/iss1/art5/

Collings, J. B., Wollkind, D. J., 1990. A global analysis of a temperaturedependent model system for a mite predator-prey interaction. SIAM J. Appl. Math. 50 (5), 1348-1372. 
Costamagna, A. C., Landis, D. A., 2006. Predators exert top-down control of soybean aphid across a gradient of agricultural management systems. Ecol. Appl. 16, 1619-1628.

Deffuant, G., Chapel, L., Martin, S., 2007. Approximating viability kernels with support vector machines. IEEE T. Automat. Contr. 52 (5), 933-937.

Doyen, L., Saint-Pierre, P., 1997. Scale of viability and minimum time of crisis. Set-Valued Anal. 5, 227-246.

Fuentes, R. E., 1993. Scientific research and sustainable development. Ecol. Appl. 3, 576-577.

Gunderson, L., Holling, C., 2002. Panarchy : Understanding Transformations in Human and Natural Systems. Island Press, Washington.

Jackson, T., 2007. Sustainable consumption. In: Atkinson, G., Dietz, S., Neumayer, E. (Eds.), A Handbook of Sustainable Development. Edward Elgar, Cheltenham, pp. 254-268.

Lacitignola, D., Petrosillo, I., Cataldi, M., Zurlini, G., 2007. Modelling socioecological tourism-based systems for sustainability. Ecol. Model. 206, 191204.

Lehtonen, M., 2004. The environmental-social interface of sustainable development: capabilities, social capital, institutions. Ecol. Econ. 49 (2), 199-214.

Levin, S. A., 1993. Science and sustainability. Ecol. Appl. 3, 545-546. 
Ludwig, D., Walker, B., Holling, C. S., 1997. Sustainability, stability, and resilience. Conserv. Ecol. 1 (1), 7.

URL http://www . consecol .org/vol1/iss1/art7/

Martin, S., 2004. The cost of restoration as a way of defining resilience: a viability approach applied to a model of lake eutrophication. Ecol. Soc. $2(9), 8$.

URL http://www . ecologyandsociety.org/vol9/iss2/art8

Martinet, V., Doyen, L., 2007. Sustainability of an economy with an exhaustible resource : A viable control approach. Resour. Energy Econ. 29 (1), 17-39.

Martinet, V., Thbaud, O., Doyen, L., 2007. Defining viable recovery paths toward sustainable fisheries. Ecol. Econ. 62 (2), 411-422.

Martinet, V., Thbaud, O., Rapaport, A., 2010. Hare or tortoise? trade-offs in recovering sustainable bioeconomic systems. Environ. Model. Assess. $15(6), 503-517$.

May, R., 1977. Thresholds and breakpoints in ecosystems with a multiplicity of stable states. Nature 269, 471-477.

Melbourne-Thomas, J., Johnson, C. R., Fung, T., Seymour, R. M., Chrubin, L. M., Arias-Gonzlez, J. E., Fulton, E. A., 2011. Regional-scale scenario modeling for coral reefs: a decision support tool to inform management of a complex system. Ecol. Appl. 21, 1380-1398.

Munasinghe, M., 1993. Environmental economics and sustainable development. World Bank, Washington, DC. 
Neubert, M. C., Caswell, H., 1997. Alternatives to resilience for measuring the responses of ecological systems to perturbations. Ecology 78 (3), 653665.

Perrings, C., 2006. Resilience and sustainable development. Environ. Devel. Econ. 11, 417-427.

Petschel-Held, G., Schellnhuber, H.-J., Bruckner, T., Toth, F., Hasselmann, K., 1999. The tolerable windows approach: Theoretical and methodological foundations. Climatic Change 41 (3-4), 303-331.

Solow, R., 1974. Intergenerational equity and exhaustible resources. Rev. Econ. Stud. 41, 29-45. 


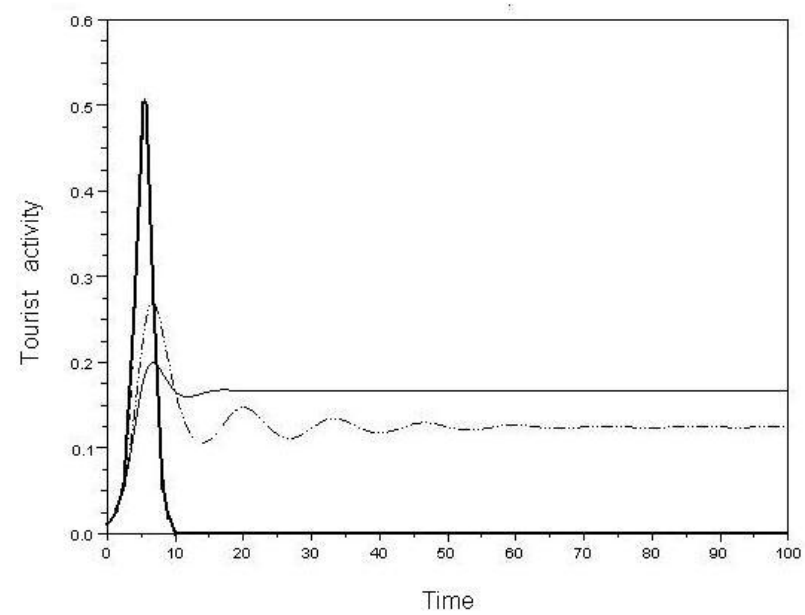

Figure 1: Scenarios of tourism development with maximal time 100. All these diagrams are obtained with the model (1). The parameter values are the same as in Casagrandi and Rinaldi (2002) with $a=6, \epsilon=0.01$ for plain line, $\epsilon=0.1$ for dotted line and $\epsilon=0.45$ for bold curve. The initial point of all curves is $(T=0.01$, $E=1, C=0.01)$ 


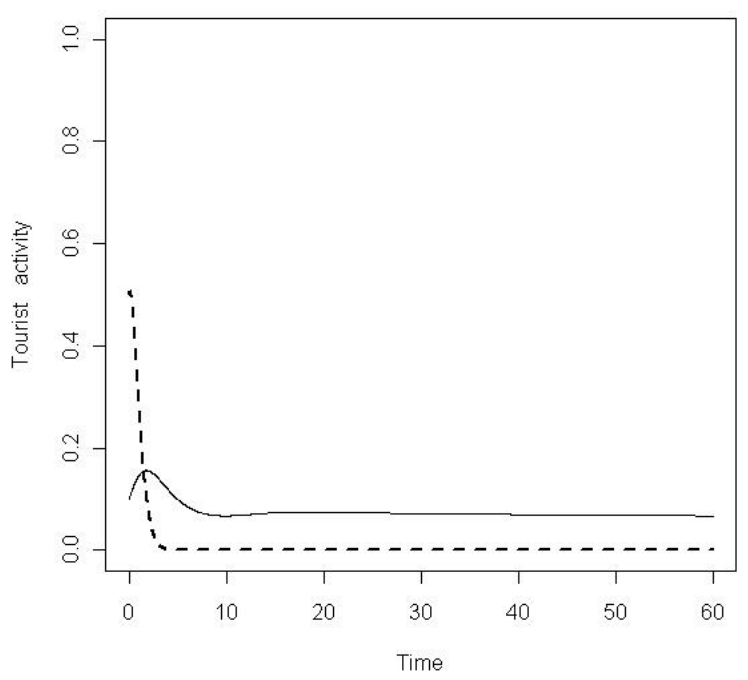

Figure 2: Scenarios of tourism development with model (1), with $a=6.8$ and $\epsilon=0.1$. Two equilibria are stable: The strictly positive one and $(0,1,0)$. The plain curve seems to converge toward the strictly positive equilibrium, its initial point $(0.1,1,0.1)$ belongs to the attraction basin of the strictly positive equilibrium. The dotted bold curve seems to converge toward 0 , its initial point $(0.5,0.5,0.5)$ belongs to the attraction basin of the equilibrium $(0,1,0)$. 


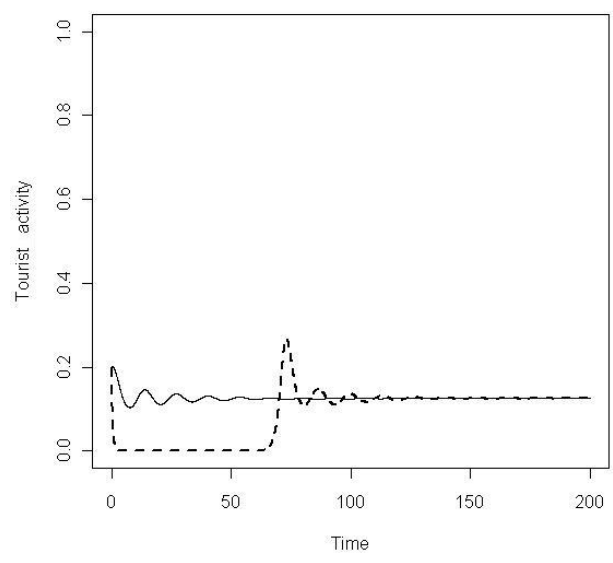

(a)

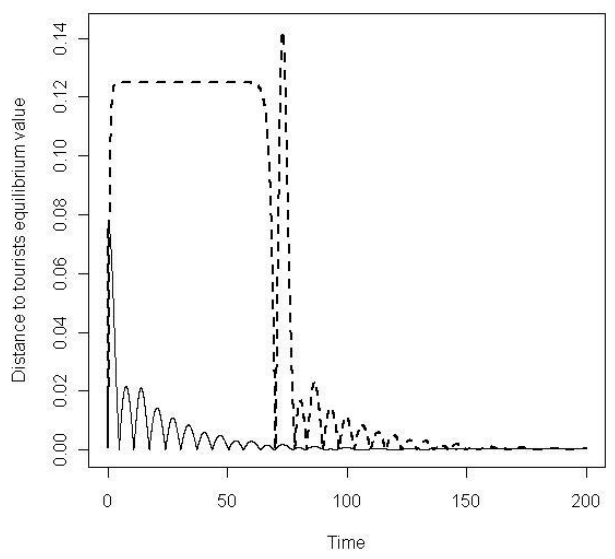

(b)

Figure 3: (a) Scenarios of tourism development with model (1), with $a=6$ and $\epsilon=$ 0.1. The strictly positive equilibrium is the only stable one. (b) Distance between the tourist activity and the attractor value. From initial point $(0.2,0.6,0.1)$, the maximum distance to the tourist equilibrium value (plain line) is the initial point. From initial point $(0.2,0.2,0.2)$ (in dotted line) it is not the case. 


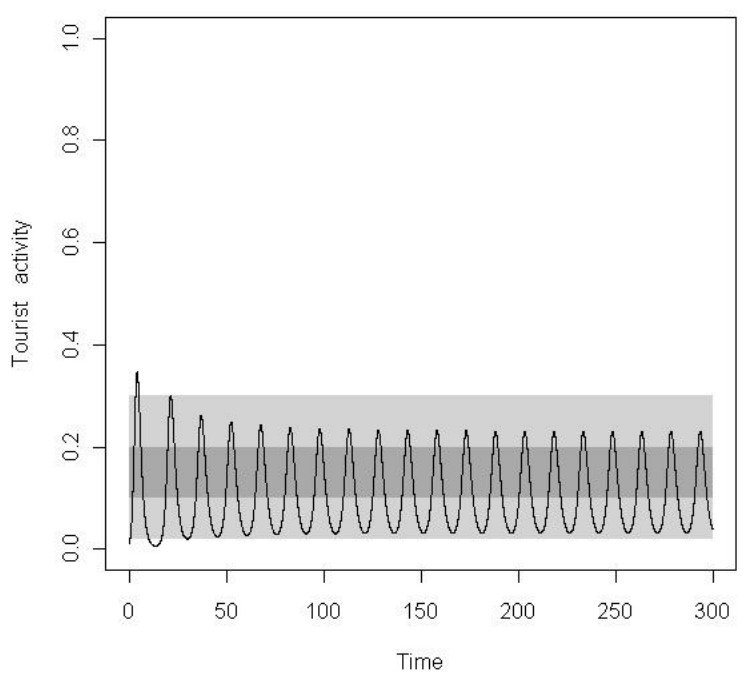

Figure 4: Scenarios of tourism development with maximal time 300. The parameter values are the same as in figure 1 with $a=6$ and $\epsilon=0.13$. The attractor is a limit cycle. The initial point is $(T=0.01, E=1, C=0.1)$. The dark gray area corresponds to $T \in[0.1 ; 0.2]$, the light gray area to $T \in[0.02 ; 0.3]$. 


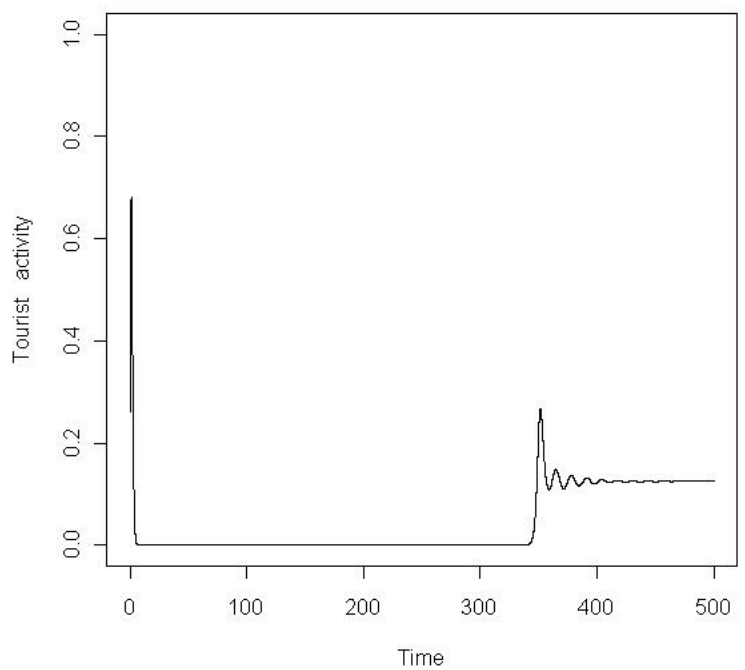

Figure 5: Scenarios of tourism development with maximal time 500. The parameter values are the same as figure 1 with $a=6$ and $\epsilon=0.1$. The initial point is $(T=0.26, E=1, C=0.26)$. 


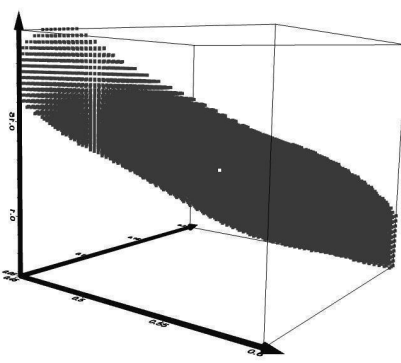

(a)

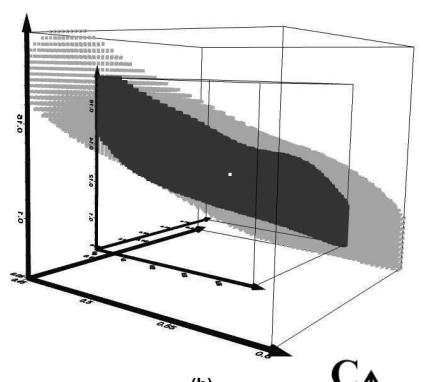

(b)

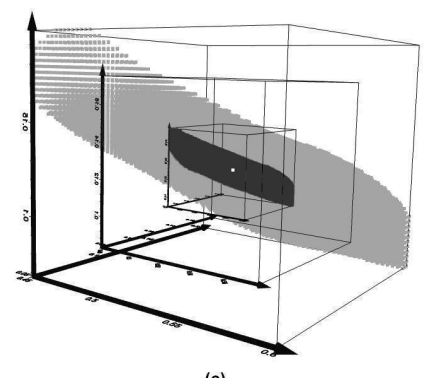

(c)

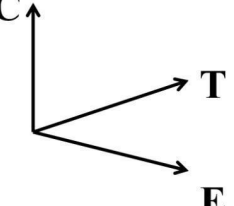

E

Figure 6: The parameter values are the same as in figure 1 with $a=6$ and $\epsilon=0.1$. Attractor is $(\bar{T} \approx 0.125, \bar{E} \approx 0.526, \bar{C} \approx 0.125)$. Dark areas represent the viability kernel for dynamics (1) and constraint set $K=[\bar{T}-\Delta ; \bar{T}+\Delta] \times[\bar{E}-\Delta ; \bar{E}+\Delta] \times$ $[\bar{C}-\Delta ; \bar{C}+\Delta], \Delta=0.075$ for diagram (a), $\Delta=0.05$ for diagram (b) and $\Delta=$ 0.025 for diagram (c). In light gray the largest viability kernel. 


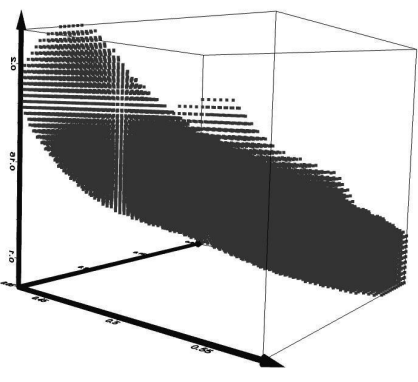

(a)

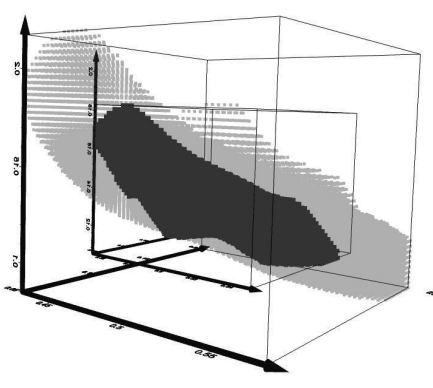

(b)

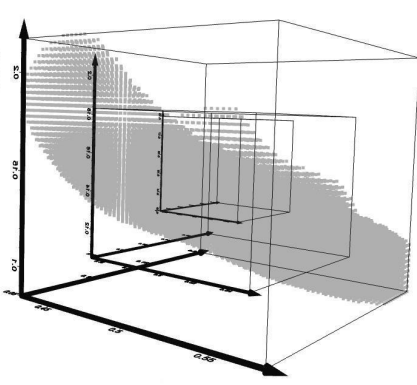

(c)

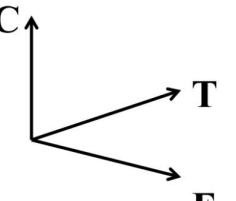

$\mathbf{E}$

Figure 7: The parameter values are the same as in figure 1 with $a=6$ and $\epsilon=0.13$, the attractor is a limit cycle in that case. Dark areas represent the viability kernel for dynamics (1) and constraint set $K=[\bar{T}-\Delta ; \bar{T}+\Delta] \times[\bar{E}-\Delta ; \bar{E}+\Delta] \times$ $[\bar{C}-\Delta ; \bar{C}+\Delta]$, with $(\bar{T} \approx 0.125, \bar{E} \approx 0.526, \bar{C} \approx 0.125) . \Delta=0.075$ for diagram (a), and $\Delta=0.05$ for diagram (b). With $\Delta=0.025$ in diagram (c), the viability kernel is empty. In light gray the largest viability kernel. 


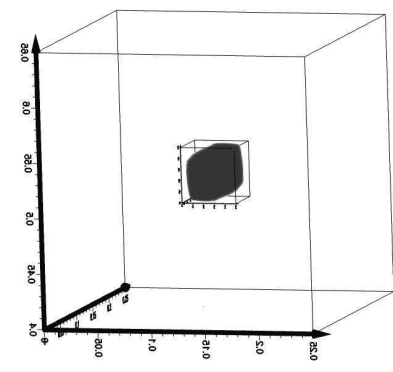

(a)
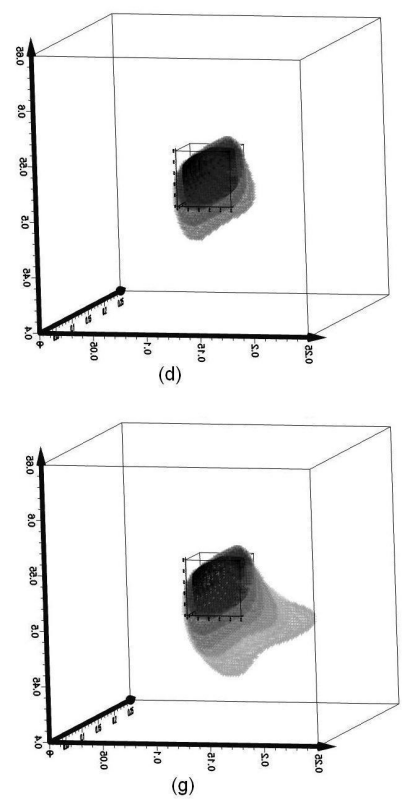
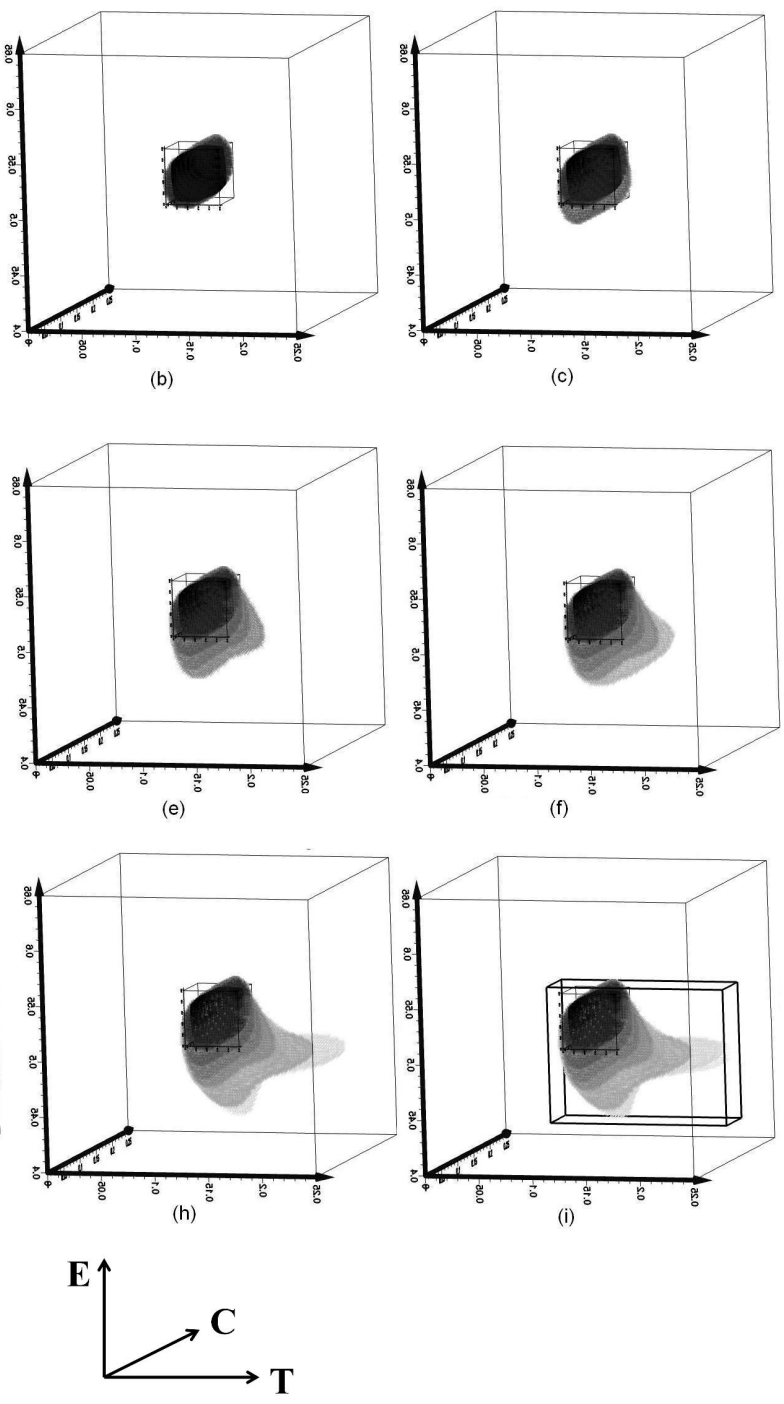

Figure 8: (a) In black, the constraint set and viability kernel displayed in figure 6 (c). (b)... (i) The shade of gray represents the successive level sets of the capture basins of the viability kernel for eight increasing reaching time $t=10, \ldots, t=80$. 

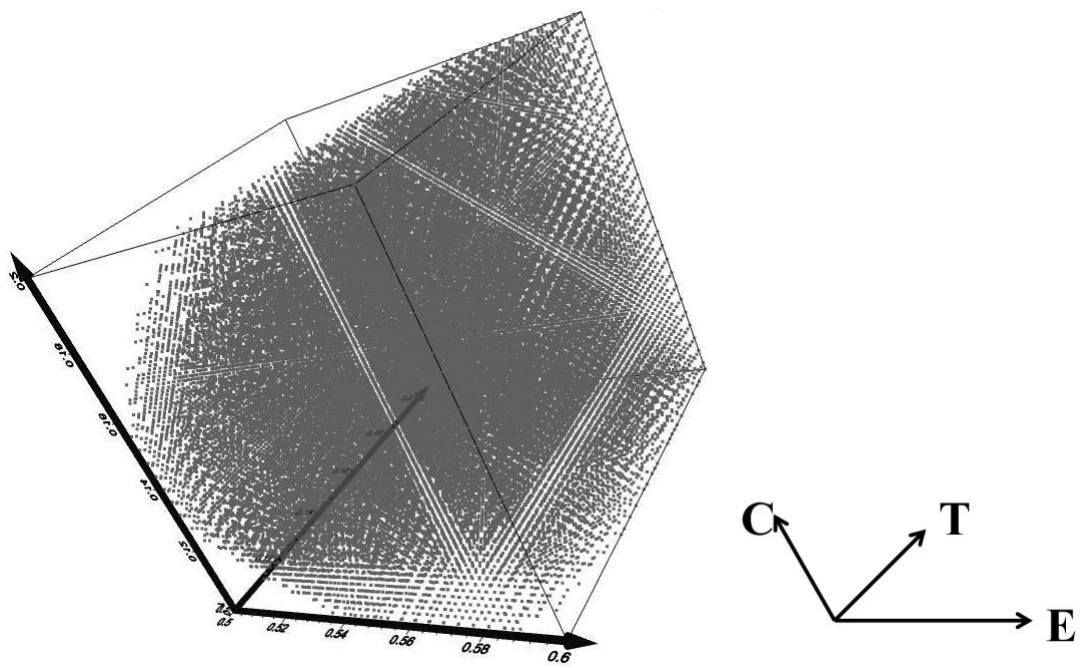

Figure 9: The dynamics are the dynamics of model (6). The parameters are the same as figure 1 with $\underline{\epsilon}=0.01, \bar{\epsilon}=0.3, \underline{a}=6$ and $\bar{a}=8$. The constraint set is $\{(T, E, C) \in[0.1 ; 0.2] \times[0.5 ; 0.6] \times[0.1 ; 0.2]\}$. The viability kernel is colored gray. 


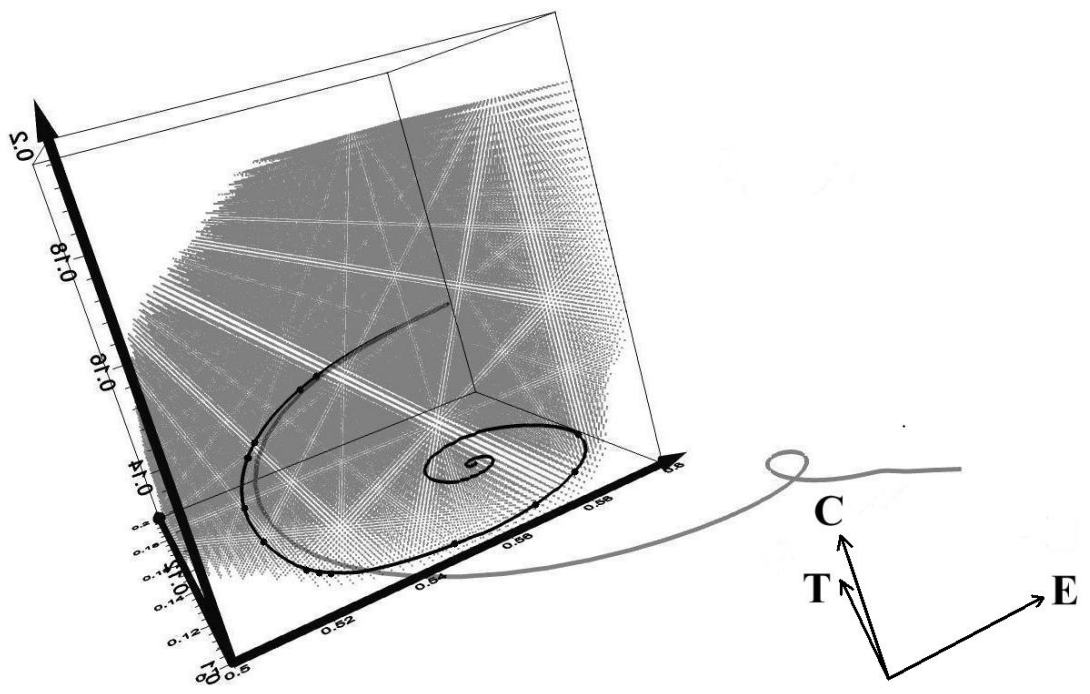

Figure 10: The trajectories of two evolutions starting from point $(T=0.15, E=$ $0.58, C=0.14)$ in the viability kernel from figure 9 . The gray one is obtained with fixed control values $\epsilon=0.01$ and $a=6$. The black one is governed by a viable control function, the points drawn on this trajectory correspond to positions where the control value changes. 


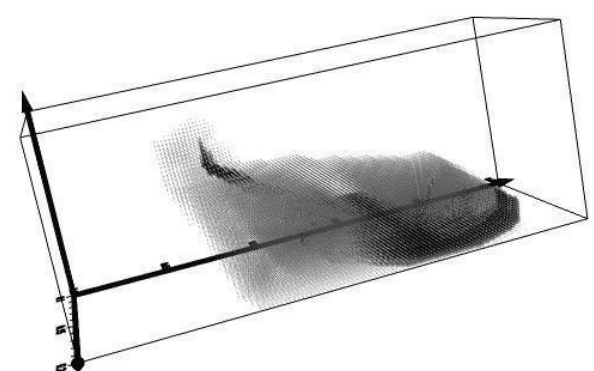

(a)

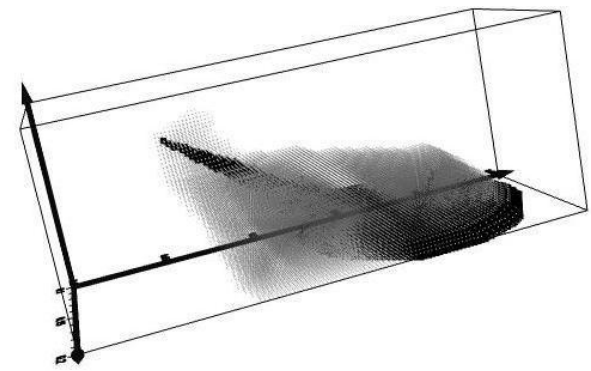

(b)

Figure 11: In gray, the viability kernel of the controlled model (6), with constant control $a=6$ and with constraint space $\{(T, E, C) \in[0.1 ; 0.2] \times[0.4 ; 0.65] \times$ $[0.1 ; 0.2]\}$. All other parameter values are the same as in figure 1 . It represents $19.2 \%$ of the constraint space. (a) In black the viability kernel with constant control $\epsilon=0.05$ (5.6\% of the constraint space). (b) In black the viability kernel with constant control $\epsilon=0.1$ (6.1\% of the constraint space). The viability kernel is empty with constant control $\epsilon=0.15,0.2,0.25$ or 0.3 . 